\title{
On the Use of UTD-Based Models for RF Path Loss Prediction Due to Diffraction on a Forest-Covered Ridge
}

\author{
Felipe M. da Costa $^{1}$ (D), Luis A. R. Ramirez ${ }^{2}$ (D), Maurício H. C. Dias ${ }^{3}$ (D) \\ ${ }^{1}$ Instituto Militar de Engenharia, Pç General Tibúrcio, 80, Rio de Janeiro, RJ, Brazil, \\ felmacedo@gmail.com \\ ${ }^{2}$ Centro Universitário Gama e Souza, Avenida Fernando Mattos, 48, Rio de Janeiro, RJ, Brazil, \\ luis.ramirez@gamaesouza.edu.br \\ ${ }^{3}$ Centro Federal de Educação Tecnológica Celso Suckow da Fonseca, Av Maracanã, 229, \\ Rio de Janeiro, RJ, Brazil, mauricio.dias@cefet-rj.br
}

\begin{abstract}
Irregular terrains covered with forest vegetation represent a challenging scenario for radio planning. A case of particular interest is the one where a forest-covered high hill or mountain is interposed to the link, for which typical diffraction loss models usually apply as good approximations, even disregarding the vegetation influence. Pragmatic approaches to incorporate the forest contribution, such as adding a clutter height, usually improve accuracy a little further. In this scope, this paper assesses the performance of some models based on the Uniform Theory of Diffraction (UTD) for RF path loss prediction in such ridges. Special attention goes to a hybrid model in which the forest is a uniform layer over a wedge that represents the ridge, and its influence is incorporated into the diffraction coefficient. The path loss predictions are compared with measurements from a mountainous region of the USA, and the statistical adherence of the models to the measured data is discussed. Overall, the slightly better performance of the models which incorporate the vegetation influence was confirmed, the hybrid model performing the best for frequencies below $910 \mathrm{MHz}$.
\end{abstract}

Index Terms - UHF propagation, Uniform Theory of Diffraction, vegetation, VHF radio propagation.

\section{INTRODUCTION}

Path loss predictions are essential for radio planning. Within this scope, there are some particular challenging scenarios that impose extra care and effort to such task, as they incorporate characteristics not fully covered by the mostly adopted prediction models. A representative example of this class of special scenarios is given by hilly or mountainous terrains covered by forest vegetation [1]

A relevant case that usually arises in that scenario is the one represented by a radio link in which a single dominant forest-covered high ridge is interposed between the transceivers. In this case, the summit usually presents hundreds of meters of altitude, while the forest layer cover measures 10-30 m high. Besides free-space loss, the radio signal is mainly degraded by diffraction on the ridge. There are many models to calculate such diffraction, from the classical knife-edge [2], through geometrical theory of diffraction [3]-[4], among others [5]-[6]. 
The diffraction loss in that scenario is usually computed disregarding the forest layer contribution. Such simplification leads to less accurate path loss predictions, as usually confirmed by measurements. A pragmatic approach to compensate such inaccuracy is the addition of a vegetation clutter height to calculate the diffraction loss, as indicated in [7]-[9], for instance. Other straightforward possibility is to add a vegetation clutter loss, using analytical models such as the ones recommended in [10]. On the other hand, more elaborate methods attempt to blend vegetation attenuation models with integral equation models that deal with the terrain height variations, as seen in [11]-[12].

In this scope, this paper addresses the use of UTD-based wedge diffraction models to calculate the RF path loss in such scenario. The original UTD model [4] and Luebbers' UTD heuristical version that accounts for finite permittivity and conductivity of the terrain [5] were chosen for this analysis. Luebbers' model is also assessed with two modifications. First, adopting the pragmatic clutter height approach previously mentioned [7]. Second, computing the Fresnel reflection coefficient taking into account the presence of a lossy uniform forest layer above the ridge [13]. Special attention goes to this second variant of Luebbers' model, which is called hereinafter "hybrid" model for ease of identification. The models performance was assessed, for VHF and UHF frequencies, computing the path loss for a number of radio links in the coniferous-covered mountain landscape of Boulder, Colorado (USA). The predictions were compared with available measurements, carried out in that site.

The paper is organized as follows. Section II explains the rationale behind the hybrid model, which is detailed in Section III. The methodology adopted to assess the UTD models is explained next, in Section IV. The obtained comparison results are presented and discussed in Section V, pointing out the statistical performance of the models. Section VI concludes the paper.

\section{HYBRID MODEL RATIONALE}

The hybrid model is based on two distinct facets of the target propagation problem: diffraction by a single dominant ridge and attenuation due to vegetation. Regarding the first one, a choice for the diffraction by a wedge representation was made. A wedge-shaped obstacle with its faces ( 0 and $n)$, as well as its relevant geometric parameters are shown in Fig. 1.

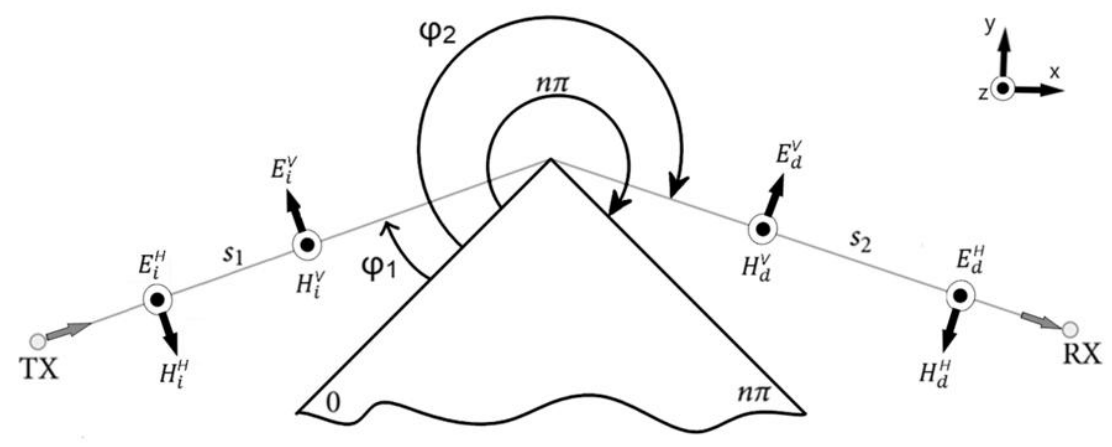

Fig. 1. Obstruction modeled as a wedge with its geometric parameters and the components of the incident and diffracted magnetic $(H)$ and electric $(E)$ fields for vertical and horizontal polarization.

The analysis of diffraction by modeling the obstacle as a wedge has been the subject of a few classical works. The first is known as Geometrical Theory of Diffraction (GTD), by Keller [3]. His 
approach presented a convergence problem in regions close to shadow boundaries (incident and reflection), which was corrected by Kouyoumjian and Pathak in their widely known UTD [4]. In both UTD and GTD, the wedge faces are perfect electrical conducting obstacles. A decade later, Luebbers heuristically incorporated reflection coefficients to the diffracted field equation, in order to better model dielectric wedges in radio propagation problems [5]. Further investigation on this case of diffraction on a lossy dielectric wedge has been conducted by others since then. In [14], a different set of asymptotic approximations was derived for the total electric field scattered around a right-angled lossy dielectric wedge at normal incidence, leading to better agreement with measurements than Luebbers' model in a few particular observation angle ranges. An issue regarding the discontinuity of the total field as a function of the angle of observation was addressed in [15], adding two new terms to the diffraction coefficient in order to correct that. In [16], Holm heuristically extended Luebbers' diffraction coefficient, providing a more accurate solution for observation points deeper in the shadow region, where the previous one supposedly fails. Other slightly different approaches on the subject are found in [17]-[19], but the significance of Luebbers' model for the radiowave propagation context remains strong, as it is widely referenced in the pertinent literature, as in [20] for instance. Actually, its acceptance is such that the model was included in the ITU-R recommendation on attenuation by diffraction in its seventh release (2001) [21], and kept up to the most recent revision (2019) [6].

In all the 2D GTD-based models, the electric field detected at the receiving point (RX) is defined according to:

$$
E_{R X}=E_{0} \cdot \frac{e^{-j k s_{1}}}{s_{1}} \cdot D \cdot \sqrt{\frac{s_{1}}{s_{2}\left(s_{1}+s_{2}\right)}} \cdot e^{-j k s_{2}},
$$

where $E_{0}$ is the relative source amplitude, $s_{1}$ is the distance from the transmission point (TX) to the diffraction point, $s_{2}$ is the distance from the diffraction point to the receiving point, $k$ is the wave number and $D$ is the diffraction coefficient, which is dependent on the specific diffraction model.

On its turn, the vegetation was modeled as a homogeneous, isotropic and lossy block, as done by Tamir [22]. In his approach, the forest layer is essentially defined by its average electrical properties (relative permittivity and conductivity) and its height. Typical values of such parameters for different kinds of forests are found in [23]. Tamir's analysis was actually limited to HF and VHF radio propagation within forests, where the lateral wave rises as the dominant propagation mechanism. A related work successfully extended the use of this lateral wave model to UHF frequencies, in order to account for the vegetation effects in the receiver near range [24]. In the present work, the forest is basically a thin layer covering the wedge, with the incident wave coming from above the forest, thus not inducing any lateral wave. Since the major propagation mechanism is the diffraction at the top of that cover, what really matters here is how the vegetation will affect that diffraction. Therefore, the single lossy isotropic homogeneous layer hypothesis was also assumed to be reasonable enough for UHF frequencies here. 


\section{HYBRID MODEL DESCRIPTION}

The hybrid model considers the wedge as composed of two layers of homogeneous and isotropic materials: an inner layer representing the ground material; and a superimposed layer of forest vegetation. Some geometric parameters required for the model are indicated in Fig. 2. Other geometric parameters are also indicated in Fig. 3.

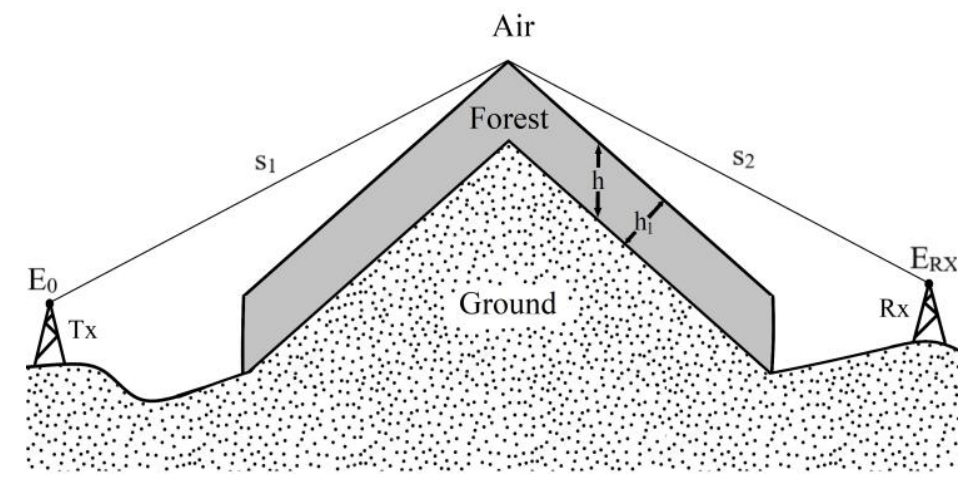

Fig. 2. Two-layer wedge-shaped obstacle (ground and vegetation) with distances, heights and points of transmission and reception.

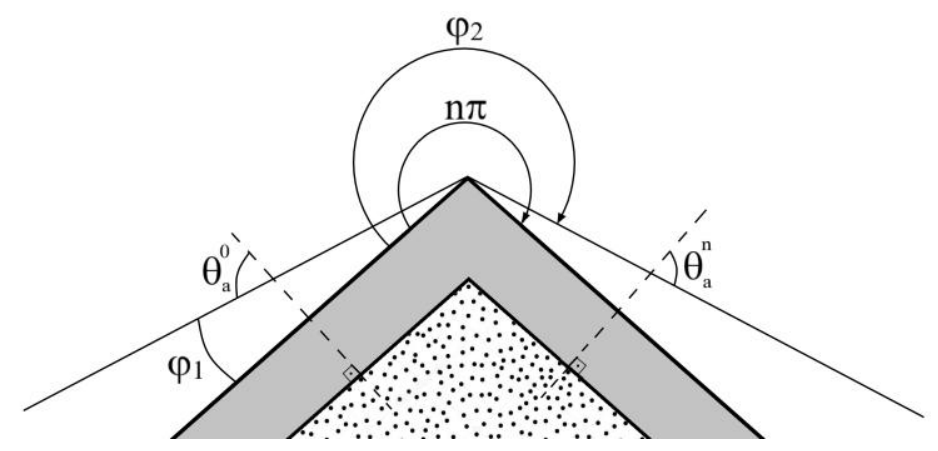

Fig. 3. Zoomed version of Fig. 2 with other important geometric parameters required by the model.

In the hybrid model, the diffraction coefficient is essentially the same as the one presented by Luebbers [5]. The difference relies on the reflection coefficients. The diffraction coefficient is, thus, given by [13]:

$$
\begin{aligned}
& D_{v e g}^{\perp}=\frac{-e^{-j \pi / 4}}{2 n \sqrt{2 \pi k}} \cdot\left\{\frac{\cot \left(\pi+\left(\varphi_{2}-\varphi_{1}\right)\right)}{2 n} \cdot F\left(k L a^{+}\left(\varphi_{2}-\varphi_{1}\right)\right)+\frac{\cot \left(\pi-\left(\varphi_{2}-\varphi_{1}\right)\right)}{2 n} \cdot F\left(k L a^{-}\left(\varphi_{2}-\varphi_{1}\right)\right)\right.
\end{aligned}
$$

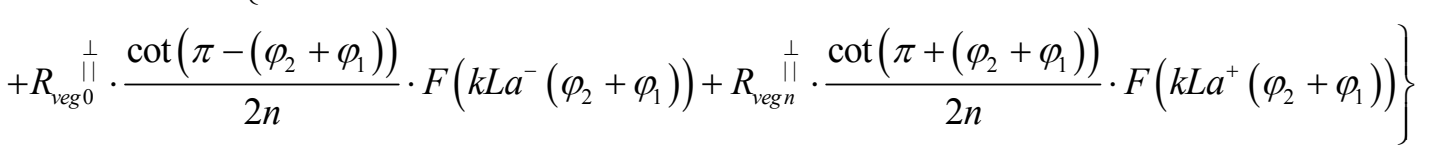

where $\varphi_{1}$ and $\varphi_{2}$ are the angles of incidence and diffraction, respectively, and $n$ is the wedge aperture factor. $F(x)$ is the Fresnel transition function, given by:

$$
F(x)=2 j \sqrt{x} e^{j x} \int_{\sqrt{x}}^{\infty} e^{-j \tau^{2}} d \tau,
$$

with argument $L$ and subfunction $a^{ \pm}(\beta)$, given respectively by [4]:

$$
L=\frac{s_{1} \cdot s_{2}}{s_{1}+s_{2}},
$$




$$
a^{ \pm}(\beta)=2 \cos ^{2}\left(\frac{2 n \pi N^{ \pm}-\beta}{2}\right)
$$

In (5), $\beta=\varphi_{2} \pm \varphi_{1}$, whereas $N^{ \pm}$is defined as the closest integer that satisfies [4]:

$$
N^{ \pm}=\frac{\beta \pm \pi}{2 n \pi} .
$$

For the scenario analyzed in this work, an expression for the reflection coefficient that incorporates the mechanisms of intra-layer reflection and refraction and oblique incidence in the interfaces is required [25]. The geometric representation for three layers and oblique incidence is illustrated by Fig. 4.

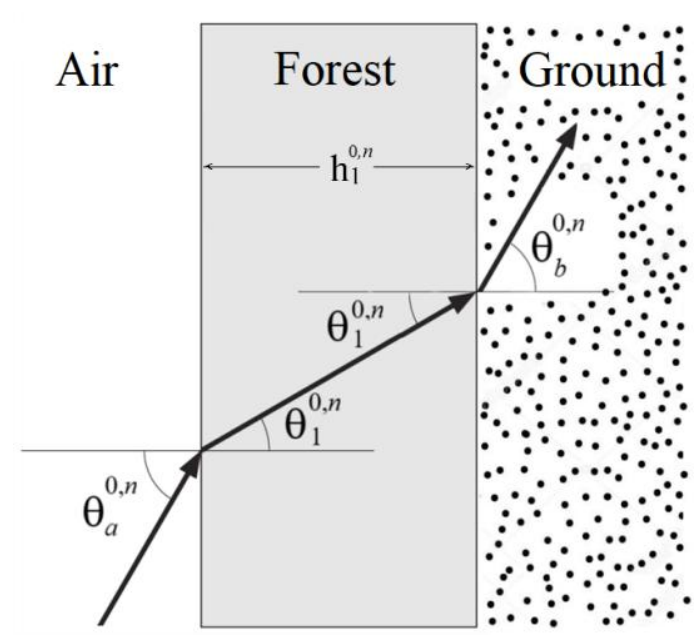

Fig. 4. Geometric representation of the successive refraction for oblique incidence with the angles referring to the faces 0 and $n$ of the wedge in each of the three layers.

The reflection coefficient will be different according to the polarization (parallel $\|$, or perpendicular $\perp$ ) and the face of the wedge $(0$ and $n)$. They are given by:

$$
\begin{aligned}
& R_{v e g} \stackrel{\perp}{\perp}=\frac{\rho_{10}^{\perp}+\rho_{20}^{\perp} e^{-2 j k_{1} h_{1 T}^{0}}}{1+\rho_{10}^{\perp} \rho_{20}^{\perp} \cdot e^{-2 j k_{1} h_{1 T}^{0}}}, \\
& R_{v e g n}^{\perp}=\frac{\rho_{1 n}^{\perp}+\rho_{2 n}^{\perp} e^{-2 j k_{1} h_{1 T}^{n}}}{1+\rho_{1 n}^{\perp} \rho_{2 n}^{\perp} \cdot e^{-2 j k_{1} h_{1 T}^{n}}}, \\
& R_{\text {veg } 0}=-\frac{\rho_{10}^{\|}+\rho_{20}^{\|} e^{-2 j k_{1} h_{1 T}^{0}}}{1+\rho_{10}^{\|} \rho_{20}^{\|} \cdot e^{-2 j k_{1} h_{1 T}^{0}}}, \\
& R_{v e g n}^{\|}=-\frac{\rho_{1 n}^{\|}+\rho_{2 n}^{\|} e^{-2 j k_{1} h_{1 T}^{n}}}{1+\rho_{1 n}^{\|} \rho_{2 n}^{\|} \cdot e^{-2 j k_{1} h_{1 T}^{n}}},
\end{aligned}
$$

where $k_{1}$ is the wave number in the intermediate medium (forest layer), given by (11), and $\varepsilon_{\mathrm{c}}$ is the complex permittivity of the medium, given by (12).

$$
k_{1}=\omega \sqrt{\mu_{0} \varepsilon_{c}}
$$




$$
\varepsilon_{c}=\varepsilon_{r} \varepsilon_{0}-j \frac{\sigma}{\omega}
$$

In (11) and (12), $\varepsilon_{0}$ and $\mu_{0}$ are, respectively, the permittivity and permeability of the vacuum, $\sigma$ is the forest layer conductivity $(\mathrm{S} / \mathrm{m}), \varepsilon_{r}$ is the relative permittivity of the forest layer, and $\omega$ is the angular frequency $(\mathrm{rad} / \mathrm{s})$. In (7)-(10), $h_{1 T^{0, n}}$ is the transverse thickness of the intermediate medium, given by (13).

$$
h_{1 T}^{0, n}=h_{1}^{0, n} \cos \left(\theta_{1}^{0, n}\right)
$$

It is worth noting that $h_{1}^{0, n}$, which represents the thickness of the forest layer, is different from $h$, which in this case represents the height of the trees. In this model, $h$ is the same in both sides of the wedge. Therefore, $h_{1}^{0} \neq h_{1}^{n}$ for asymmetric wedges, as illustrated by Fig. 5 .

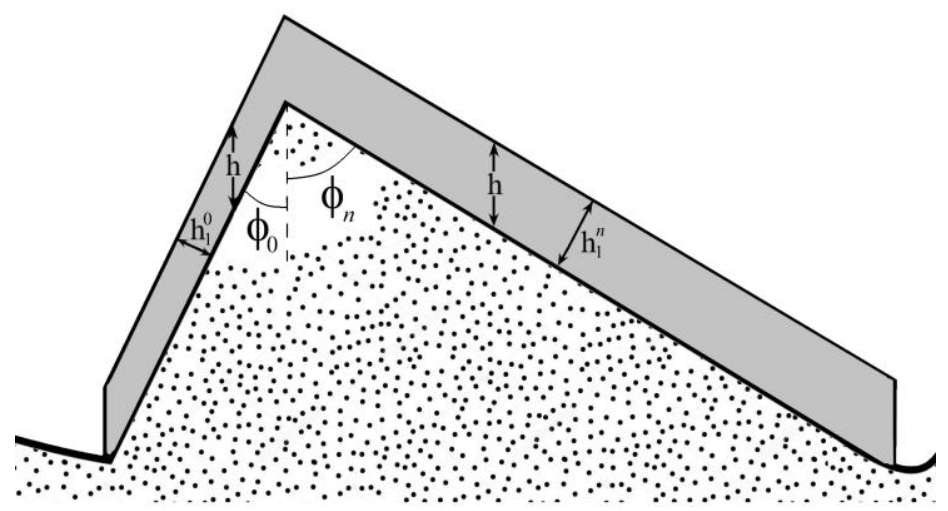

Fig. 5. Asymmetric wedge with internal angles, height of trees $(h)$ and height of layer on each face $\left(h_{1}^{0, n}\right)$.

In (7)-(10), $\rho_{1}$ is the Fresnel reflection coefficient at the air-vegetation interface and $\rho_{2}$ is the same Fresnel reflection coefficient, but at the vegetation-ground interface. These coefficients, according to the polarization $(\|$ and $\perp)$ and wedge face $(0$ and $n)$ are given by:

$$
\begin{gathered}
\rho_{10, n}^{\perp,||}=\frac{\eta_{T 1_{0, n}}^{\perp, \mid}-\eta_{T a_{0, n}}^{\perp, \mid}}{\eta_{T 1_{0, n}}^{\perp, \mid}+\eta_{T a_{0, n}}^{\perp, \mid}}, \\
\rho_{20, n}^{\perp,||}=\frac{\eta_{T b_{0, n}}^{\perp, \mid}-\eta_{T 1_{0, n}}^{\perp, \mid}}{\eta_{T b_{0, n}}^{\perp, \mid}+\eta_{T 1_{0, n}}^{\perp, \mid}},
\end{gathered}
$$

where all the $\eta_{T}$ are transverse wave impedances given, according to the respective polarizations, layers, incidence angles and wedge faces, by (16) and (17). In these, $\eta_{a, 1, b}$ is given by (18). In (16)(18), the subscripts $a, 1$ and $b$ stand for the air, forest and ground layers, respectively, as seen in Fig. 4.

$$
\begin{gathered}
\eta_{T_{a, 1, b}, n}^{\perp}=\frac{\eta_{a, 1, b}}{\cos \left(\theta_{a, 1, b}^{0, n}\right)} \\
\eta_{T_{a, 1, b_{0, n}}}^{\|}=\eta_{a, 1, b} \cos \left(\theta_{a, 1, b}^{0, n}\right)
\end{gathered}
$$




$$
\eta_{a, 1, b}=\sqrt{\frac{\mu_{0}}{\varepsilon_{c_{a, 1, b}}}}
$$

\section{METHODOLOGY}

In this work, the methodology adopted to evaluate the different diffraction models in the forestcovered ridge scenario was the comparison of simulated and measured path losses, for a properly chosen reference site. There was no intention, nor means, to pursue a more comprehensive comparison with more complex and numerically demanding deterministic approaches, such as the parabolic equation method [26], ray-tracing models [20] or other full-wave implementations available at propagation analysis suites [27]. Only deterministic UTD-based models were considered, for their analytical simplicity and good performance for asymptotic arguments (high distances and frequencies), as the ones expected in the analyzed scenario.

Thus, in order to carry out the intended analysis, the mountain landscape of Boulder, Colorado (USA) was chosen as reference site. An extensive report of path loss measurements in that area is available in a compilation provided by the Institute for Telecommunication Sciences (ITS) [28]. The documentation is divided in four volumes and contemplates different regions, all in the state of Colorado. The part II of the compilation was the one chosen for this work, since it included the largest number of links with features more closely related to the target scenario of this paper.

The measurements were carried out keeping a fixed receiver spot in which the antenna was horizontally polarized and its height varied from 1 to $13 \mathrm{~m}$, at the frequencies of $230,410,751,910$ and $1846 \mathrm{MHz}$, plus two other higher frequencies at SHF. Only the frequencies below $2 \mathrm{GHz}$ from this list were considered here. Regarding link distance ranges, the measurements were taken within $0.5,3,5,10,20$ and $50 \mathrm{~km}$, and two other higher distances beyond $50 \mathrm{~km}$. The ranges of the links compliant with the target scenario were $5,10,20$ and $50 \mathrm{~km}$. The heights of the transmission antennas varied according to the frequency used, being $6.6 \mathrm{~m}$ for the frequencies of 230,410 and $751 \mathrm{MHz}$ and $7.3 \mathrm{~m}$ for the frequencies of 910 and $1846 \mathrm{MHz}$.

Overall, eight links compliant with the desired scenario were picked out from the ITS compilation: two for a distance of $5 \mathrm{~km}$ (R2-5-T4, R2-5-T6), three for $10 \mathrm{~km}$ (R2-10-T1, R2-10-T3, R2-10-T5), one for $20 \mathrm{~km}(\mathrm{R} 2-20-\mathrm{T} 8)$ and two for $50 \mathrm{~km}$ (R2-50-T4, R2-50-T5-O). A satellite view of the Boulder region with the selected transmitter points and the common receiver (Rx) is given in Fig. 6.

From the part II of the compilation in [28], geographical and system data from the radio links were available to be replicated in the predictions of the analyzed models. With such information in hands, the respective 2D terrain profiles were reproduced, reading SRTM [29] elevation bases with the aid of an adaptation of a MATLAB ${ }^{\circledR}$ script intended to plot elevation maps [30]. Fig. 7 shows the 2D profile of one of those links (R2-5-T4), highlighting the diffracting wedge representing the actual ridge. 


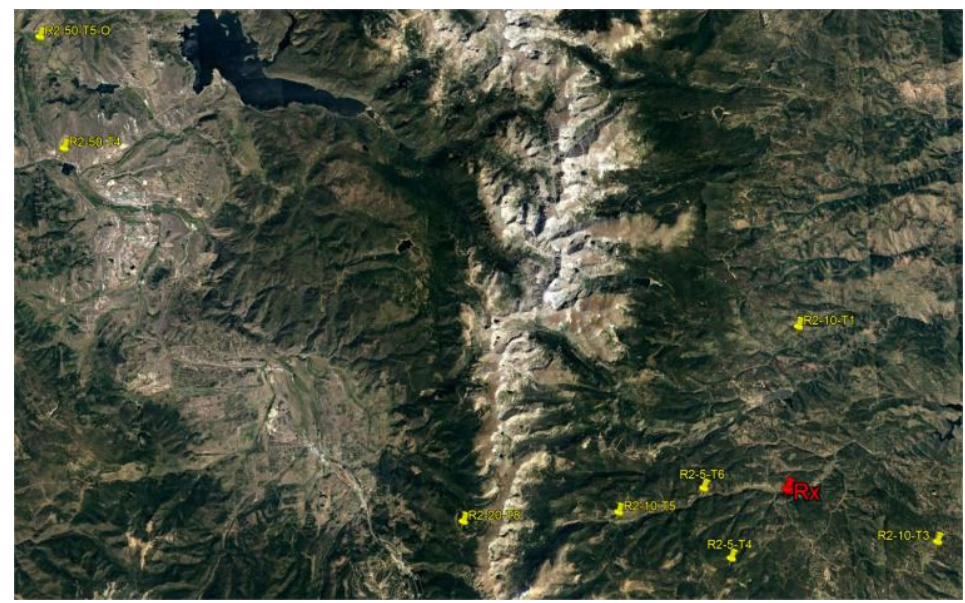

Fig. 6. Satellite view of the Boulder region chosen as reference site, containing the transmitter points and the common receiver $(\mathrm{Rx})$ of the selected ITS links.

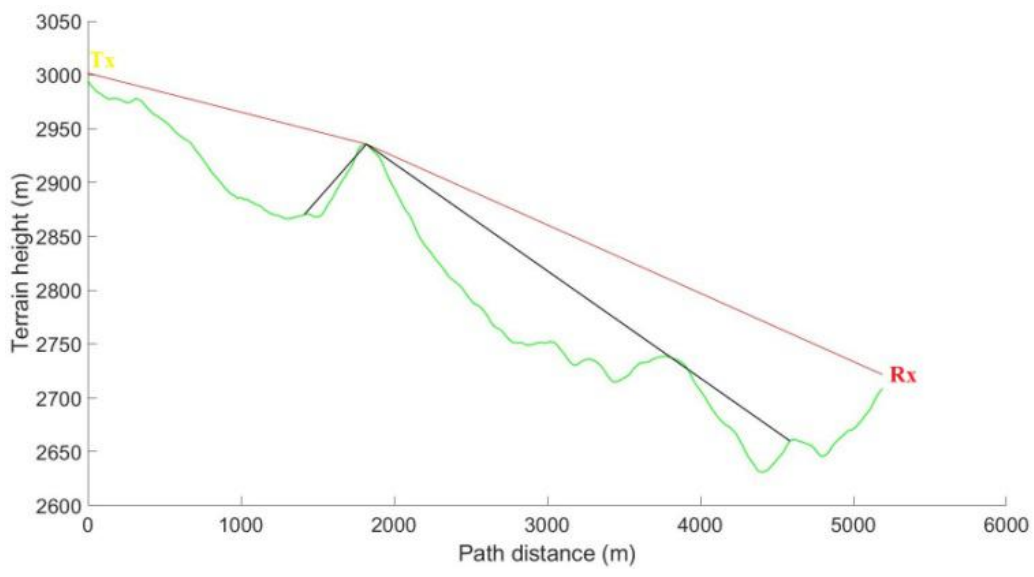

Fig. 7. Vertical terrain profile of one of the analyzed links from Boulder (R2-5-T4), highlighting the identified diffractive wedge.

Four UTD-based models were chosen to carry out the present analysis: UTD itself [4]; Luebbers' [5], disregarding vegetation; Luebbers' [5], adding a clutter height representing the vegetation (as in [7]); and Luebbers', with the hybrid adaptation described in Sections II and III (as in [13]). In all models, Earth curvature and non-homogeneity of the troposphere were considered by adopting the equivalent Earth approach, where the propagation ray is a straight line, but the Earth's radius is multiplied by a $K$ factor that is a function of the vertical gradient of the troposphere refractivity [2]. Values of the $K$ factor worldwide can be found at [31], from which $K=1.21$ (exceeded for $50 \%$ of the time) was used. All models were implemented in MATLAB ${ }^{\circledR}$ scripts, using the terrain profiles of the eight chosen links.

Apart UTD, the other three models rely on electrical properties of the terrain as well: relative permittivity $\left(\varepsilon_{r}\right)$ and conductivity $(\sigma)$. The ridge electrical parameters were estimated from pertinent references, in special [32]: $\varepsilon_{r b}=13$ and $\sigma_{b}=5 \mathrm{mS} / \mathrm{m}$. Regarding the vegetation layer, a narrow range of values was first chosen as proper estimates, based on information from [28], as well as the suggestions in [23]. A single pair of values were considered for all runs of the hybrid model: $\varepsilon_{r 1}=1.23$ and $\sigma_{1}=0.3 \mathrm{mS} / \mathrm{m}$. Furthermore, these three models need information on the forest layer 
height $(h)$. Again, information from [23] and [28] was useful to extract a narrow range of representative values, from which the height $h=12 \mathrm{~m}$ was chosen as estimate, and thus considered in all runs of the hybrid model and the Luebbers' + clutter height model.

The calculated path losses of all models were compared to the measured data, from which statistical moments of the differences were derived. For each (transmitter position, frequency) pair, there was a set of measured values, $M=\left\{m_{1}, m_{2}, \ldots, m_{N}\right\}$, with $N$ being the number of receiver heights (up to 13), and there were 4 sets of simulated path loss values for the same receiver heights, $S_{j}=\left\{s_{1}, s_{2}, \ldots, s_{N}\right\}$, $j=\{1,2,3,4\}$, one for each model, all in $\mathrm{dB}$. For these $4 S_{j}$ sets and for each (transmitter position, frequency) pair, the respective sets of absolute errors in respect to the measurements were obtained, $E_{j}=\left\{e_{j 1}, e_{j 2}, \ldots, e_{j N}\right\}$, where $e_{j i}=\left(s_{j i}-m_{i}\right)$. Then, for each available $E_{j}$ set and for each (transmitter position, frequency) pair, the respective mean absolute error (ME) was calculated according to (19), the root mean square error (RMSE) was given by (20), and the standard deviation (SD) by (21). As a result, for each of the $8 \times 5$ (transmitter position, frequency) pairs, there were ME, RMSE and SD values of the 4 models.

$$
\begin{gathered}
\bar{e}=\frac{\sum_{i=1}^{N} e_{i}}{N} \\
\bar{e}_{r m s}=\sqrt{\frac{\sum_{i=1}^{N}\left(e_{i}\right)^{2}}{N}} \\
\sigma=\sqrt{\frac{\sum_{i=1}^{N}\left(e_{i}-\bar{e}\right)^{2}}{N-1}}
\end{gathered}
$$

At last, the calculated statistics were further sorted out in terms of frequency and distance range parameterization. Regarding frequency dependence, for each frequency there were 8 different transmitter positions, and thus the averages of the corresponding $8 \mathrm{ME}$, RMSE and SD were calculated. Likewise, regarding distance dependence, for each transmitter position there were 5 different frequencies, and thus the averages of the corresponding $5 \mathrm{ME}$, RMSE and SD were calculated. In this case, a further averaging process was due for the transmitter positions that had the same distance. For instance, there were 3 different transmitter positions at $10 \mathrm{~km}$, thus the averages of the corresponding $3 \mathrm{ME}, \mathrm{RMSE}$ and SD were taken to get the statistics for that distance. The same procedure was done for the distances of $5 \mathrm{~km}$ ( 2 positions) and $50 \mathrm{~km}$ ( 2 positions).

\section{RESULTS}

Simulations of the four UTD-based models were run for the eight Boulder links, for all 13 receiver heights and at the five chosen frequencies. In order to provide a first glimpse of the order of magnitude of the analyzed values, Table I summarizes the measured path losses and the corresponding simulated losses calculated by the hybrid model, as a function of the transmitter position and frequency. Positions P1 and P2 corresponded to the $5 \mathrm{~km}$ range, P3 to P5 corresponded 
to the $10 \mathrm{~km}$ range, $\mathrm{P} 6$ was at $20 \mathrm{~km}$, and P7 and P8 were at $50 \mathrm{~km}$. Table II is analogous to Table I, as it presents the simulated path losses for two other models, Luebbers' and Lubbers' + clutter height. In both, for each (transmitter position, frequency) pair, an average of the losses at the $N$ receiver heights is shown. Fig. 8 helps to assess how close the predictions got to the measurements, taking the hybrid model as example. Fig. 8 (a) shows a plot of path loss vs. distance, in which for each distance associated to 2 or 3 transmitter positions, the average for that distance was taken. On its turn, Fig. 8 (b) shows path loss vs. frequency. In both, for each represented link, an average of the $N$ path loss values was represented.

TABLE I. AVERAGE PATH LOSSES (DB) FOR ALL LINKS - MEASUREMENTS AND HYBRID MODEL

\begin{tabular}{ccccccccccc}
\hline & \multicolumn{4}{c}{ Measurements [28] } & \multicolumn{5}{c}{ Hybrid (12 m) [13] } \\
$\begin{array}{c}\text { Frequency (MHz) } \\
\text { Position }\end{array}$ & 230 & 410 & 751 & 910 & 1846 & 230 & 410 & 751 & 910 & 1846 \\
\hline P1 & 112.8 & 122.4 & 133.6 & 138.9 & 149.5 & 112.1 & 122.7 & 130.5 & 131.0 & 141.3 \\
P2 & 123.6 & - & 146.8 & 143.8 & 165.7 & 129.2 & - & 141.6 & 152.2 & 158.7 \\
P3 & 131.3 & 144.4 & - & 159.0 & 169.8 & 134.8 & 141.1 & - & 151.9 & 160.0 \\
P4 & 138.5 & 146.8 & 168.7 & 173.3 & 181.5 & 142.4 & 161.6 & 169.3 & 163.7 & 179.2 \\
P5 & 126.8 & 142.3 & 148.0 & 157.6 & 165.4 & 130.6 & 140.4 & 149.0 & 157.5 & 162.3 \\
P6 & 152.2 & 159.0 & 173.2 & 182.3 & 185.3 & 151.0 & 160.2 & 175.4 & 182.8 & 173.9 \\
P7 & 147.5 & 160.5 & 169.8 & 174.8 & 191.0 & 152.4 & 164.1 & 167.8 & 177.9 & 186.7 \\
P8 & 143.8 & 151.8 & 173.4 & 176.6 & 189.1 & 149.8 & 164.2 & 171.3 & 174.6 & 183.4 \\
\hline
\end{tabular}

TABLE II. AVERAgE PATH LOSSES (DB) FOR ALL LINKS - LUEBBERS' AND LUEBBERS' + CLUTTER HEIGHT MODELS

\begin{tabular}{ccccccccccc}
\hline & \multicolumn{4}{c}{ Luebbers [5] } & \multicolumn{5}{c}{ Luebbers (12 m) [7] } \\
$\begin{array}{c}\text { Frequency (MHz) } \\
\text { Position }\end{array}$ & 230 & 410 & 751 & 910 & 1846 & 230 & 410 & 751 & 910 & 1846 \\
\hline P1 & 112.4 & 119.5 & 127.2 & 129.7 & 138.8 & 115.8 & 123.2 & 131.0 & 133.5 & 142.7 \\
P2 & 132.2 & - & 147.6 & 150.1 & 159.3 & 134.8 & - & 150.2 & 152.7 & 161.9 \\
P3 & 131.0 & 138.0 & - & 148.2 & 157.4 & 135.5 & 142.7 & - & 153.0 & 162.2 \\
P4 & 160.5 & 168.0 & 175.7 & 178.3 & 187.5 & 161.8 & 169.3 & 177.0 & 179.6 & 188.8 \\
P5 & 138.8 & 146.3 & 154.2 & 156.7 & 165.9 & 140.4 & 147.9 & 155.8 & 158.3 & 167.5 \\
P6 & 169.3 & 176.8 & 184.7 & 187.2 & 196.4 & 169.4 & 176.9 & 184.8 & 187.3 & 196.5 \\
P7 & 160.2 & 167.7 & 175.6 & 178.1 & 187.4 & 160.5 & 168.0 & 175.9 & 178.4 & 187.7 \\
P8 & 160.0 & 167.6 & 175.5 & 178.0 & 187.2 & 160.3 & 167.9 & 175.8 & 178.3 & 187.5 \\
\hline
\end{tabular}

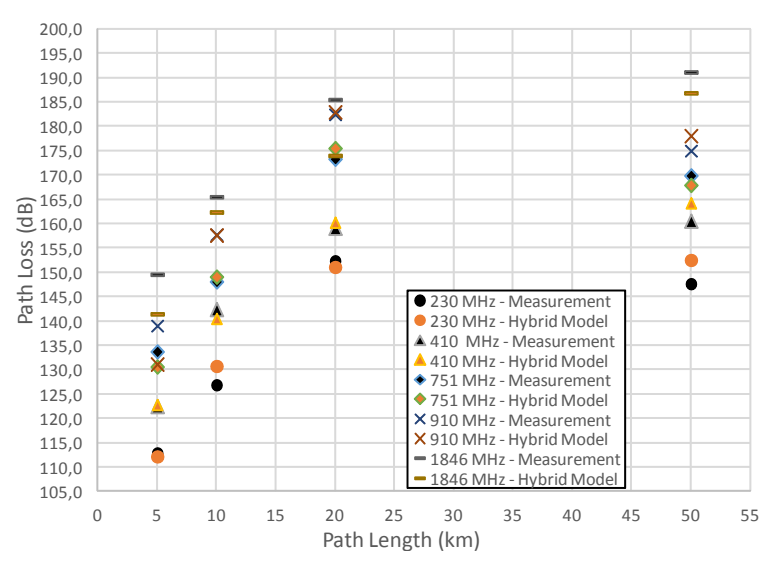

(a)



(b)

Fig. 8. Comparison between the hybrid model and measurements: (a) path loss vs. distance, (b) path loss vs. frequency. 
Following the procedures detailed at the end of Section IV, the calculated statistics results are presented in Tables III and IV, the former regarding distance, the latter frequency variation. Overall, for most results, Luebbers' approaches (all 3 variations tested) outperform UTD, as expected. The incorporation of the forest layer, either as a clutter height or by the hybrid model, mainly provides fine tuning to Luebbers' model. For the available data, it is possible to infer that the hybrid model achieved best performance for all distances and lower frequencies (below $910 \mathrm{MHz}$ ), while the clutter height model performed slightly better at higher frequencies. It is worth remarking that these results are coherent with those in [13], in which the measurements available were obtained in Rio de Janeiro, at the frequency of $563 \mathrm{MHz}$.

TABLE III. MODELS’ PERFORMANCES - DISTANCE VARIATION

\begin{tabular}{ccccc}
\hline Distance $(\mathrm{km})$ & Model & RMSE $(\mathrm{dB})$ & ME $(\mathrm{dB})$ & $\mathrm{SD}(\mathrm{dB})$ \\
\hline \multirow{3}{*}{5} & Hybrid (12m) [13] & 5.42 & -2.31 & 3.43 \\
& Luebbers (12 m) [7] & 6.35 & 1.00 & 3.43 \\
& Luebbers [5] & 6.80 & -2.21 & 3.42 \\
\multirow{2}{*}{10} & UTD [4] & 6.92 & -1.67 & 3.42 \\
& Hybrid (12m) [13] & 6.48 & 0.48 & 4.17 \\
& Luebbers (12 m) [7] & 9.67 & 6.04 & 4.40 \\
& Luebbers [5] & 9.68 & 3.63 & 4.36 \\
\multirow{2}{*}{20} & UTD [4] & 10.79 & 5.01 & 4.56 \\
& Hybrid (12m) [13] & 5.84 & -5.37 & 1.95 \\
& Luebbers (12 m) [7] & 6.90 & 5.71 & 1.94 \\
& Luebbers [5] & 6.70 & 5.39 & 1.94 \\
& UTD [4] & 11.27 & 11.00 & 1.83 \\
& Hybrid (12m) [13] & 6.11 & 2.48 & 2.49 \\
& Luebbers (12 m) [7] & 8.28 & 6.91 & 2.49 \\
& Luebbers [5] & 8.11 & 6.62 & 2.49 \\
& UTD [4] & 8.59 & 7.43 & 2.49 \\
\hline
\end{tabular}

TABLE IV. MODELS’ PERFORMANCES - FREQUENCY VARIATION

\begin{tabular}{|c|c|c|c|c|}
\hline Frequency $(\mathrm{MHz})$ & Model & RMSE (dB) & $\mathrm{ME}(\mathrm{dB})$ & $\mathrm{SD}(\mathrm{dB})$ \\
\hline \multirow{4}{*}{230} & Hybrid (12m) [13] & 6.23 & 4.46 & 3.03 \\
\hline & Luebbers $(12 \mathrm{~m})$ [7] & 12.63 & 12.21 & 2.99 \\
\hline & Luebbers [5] & 11.31 & 10.37 & 3.01 \\
\hline & UTD [4] & 12.83 & 11.98 & 3.00 \\
\hline \multirow{4}{*}{410} & Hybrid (12m) [13] & 5.73 & 3.63 & 2.61 \\
\hline & Luebbers $(12 \mathrm{~m})$ [7] & 10.18 & 9.20 & 2.60 \\
\hline & Luebbers [5] & 10.37 & 7.42 & 2.61 \\
\hline & UTD [4] & 11.93 & 9.12 & 2.60 \\
\hline \multirow{4}{*}{751} & Hybrid (12m) [13] & 3.84 & -0.54 & 2.40 \\
\hline & Luebbers (12 m) [7] & 5.69 & 4.19 & 2.77 \\
\hline & Luebbers [5] & 5.40 & 2.79 & 2.69 \\
\hline & UTD [4] & 6.93 & 4.43 & 2.92 \\
\hline \multirow{4}{*}{910} & Hybrid (12m) [13] & 6.64 & -3.54 & 4.18 \\
\hline & Luebbers $(12 \mathrm{~m})$ [7] & 6.17 & 1.06 & 4.21 \\
\hline & Luebbers [5] & 6.64 & -0.81 & 4.18 \\
\hline & UTD [4] & 7.21 & 0.69 & 4.25 \\
\hline \multirow{4}{*}{1846} & Hybrid (12m) [13] & 7.48 & -5.64 & 3.89 \\
\hline & Luebbers (12 m) [7] & 6.04 & -1.09 & 3.98 \\
\hline & Luebbers [5] & 7.17 & -2.97 & 3.97 \\
\hline & UTD [4] & 7.89 & -1.47 & 4.07 \\
\hline
\end{tabular}


Journal of Microwaves, Optoelectronics and Electromagnetic Applications, Vol. 20, No. 2, June 2021 DOI: http://dx.doi.org/10.1590/2179-10742021v20i21171

A final comment is noteworthy regarding the distinct frequency performance of the hybrid model. It was expected that the homogeneous and isotropic layer model would be better suited to lower frequency bands, because as the frequency increases, the individual components of the vegetation block will have the same order of magnitude of the respective wavelength (gradually, trunks, branches and leaves). Thus, the performance was actually consistent with theoretical expectations.

\section{CONCLUSION}

This paper discussed the use of UTD-based wedge diffraction models to calculate the RF path loss due to diffraction over forest-covered high ridges. Along with the original UTD model, Luebbers' approach was chosen, for its proven results in similar propagation scenarios. Since none of them actually incorporates the effect of the vegetation layer above the ridge, two variations of Luebbers' model were brought to the analysis. One of these simply adds a clutter height to the ridge, while the other (the hybrid model) modifies the Fresnel reflection coefficient within the diffraction coefficient equation, to account for the presence of a lossy uniform forest layer above the wedge.

The models performance was assessed computing the path loss for a number of radio links in the region of Boulder, Colorado (USA), covering distances from 5 to $50 \mathrm{~km}$, at VHF and UHF frequencies. The predictions were compared with available measurements carried out in that site.

The models comparison was performed statistically, calculating the root mean square error, mean error and standard deviation of the respective path loss predictions with its correspondent measured values. Overall, good adherence to the measurements was observed for all UTD-based models, but with UTD clearly being outperformed by all three Lubbers' versions. The two variations that incorporated the vegetation effect provided fine tuning to the results, with the hybrid model performing the best for frequencies below $910 \mathrm{MHz}$. Therefore, within the limits discussed here and when best statistical performance is desired, the hybrid model might be a suitable recommendation, under the cost of dealing with a more elaborate analytical solution in regard to the pragmatic clutter height addition usual approach.

\section{ACKNOWLEDGMENT}

This work was financed in part by the Coordenação de Aperfeiçoamento de Pessoal de Nível Superior - Brasil (CAPES) - Finance Code 001

\section{REFERENCES}

[1] F. M. Costa, L. A. R. Ramirez, M. H. C. Dias, "Analysis of ITU-R VHF/UHF propagation prediction methods performance on irregular terrains covered by forest," IET Microwaves, Antennas \& Propagation, vol. 12, no. 8, pp. $1450-1455,2018$.

[2] N. Blaunstein, C. G. Christodoulou. Radio propagation and adaptive antennas for wireless communication networks: terrestrial, atmospheric, and ionospheric, $2^{\text {nd }}$ ed. Hoboken, USA: Wiley, 2014.

[3] J. B. Keller, "Geometrical theory of diffraction," Journal of the Optical Society of America, vol. 52, no. 2, pp. 116-130, 1962.

[4] R. G. Kouyoumjian, P. H. Pathak, "A uniform geometrical theory of diffraction for an edge in a perfectly conducting surface," Proceedings of the IEEE, vol. 62, no 11, pp. 1448-1461, 1974

[5] R. J. Luebbers, "Finite conductivity uniform GTD versus knife edge diffraction in prediction of propagation path loss," IEEE Transactions on Antennas and Propagation, vol 32, no. 1, pp. 70-76, 1984 
[6] Propagation by diffraction, ITU-R P.526-15, 2019.

[7] M. Meeks, "VHF propagation over hilly, forested terrain," IEEE Transactions on Antennas and Propagation, vol 31, no. 3, pp. 483-489, 1983.

[8] Method for point-to-area predictions for terrestrial services in the frequency range $30 \mathrm{MHz}$ to $4000 \mathrm{MHz}$, ITU-R P.1546-6, 2019.

[9] A path-specific propagation prediction method for point-to-area terrestrial services in the VHF and UHF bands, ITU-R P.1812-5, 2019.

[10] Attenuation in vegetation, ITU-R P.833-9, 2016.

[11] O. Ravard, M. L. Palud, "Influence of the vegetation on a VHF radio link over an irregular terrain," in Proc. Tenth International Conf. Antennas and Propagation, Edinburgh, UK, 1997, pp. 15-18.

[12] M. L. Palud, "Propagation modeling of VHF radio channel in forest environments," in IEEE Military Communications Conference, Monterey, USA, 2004, pp. 609-614.

[13] F. M. Costa, L. A. R. Ramirez, M. H. C. Dias, "Modelo Híbrido para Perda de Propagação em Radioenlaces sobre Terrenos Irregulares com Floresta," (in Portuguese), in MOMAG $2020-19^{\circ}$ Simpósio Brasileiro de Microondas e Optoeletrônica e $14^{\circ}$ Congresso Brasileiro de Eletromagnetismo, Niterói, Brazil, 2020, pp. 1-4.

[14] C. Demetrescu, C. C. Constantinou, M. J. Mehler, "Scattering by a right-angled lossy dielectric wedge," IEE Proceedings - Microwaves, Antennas and Propagation, vol. 144, no. 5, pp. 392-396, 1997.

[15] J. F. Rouviere, N. Douchin, P. F. Combes, "Diffraction by lossy dielectric wedges using both heuristic UTD formulations and FDTD," IEEE Transactions on Antennas and Propagation, vol. 47, no. 11, pp. 1702-1708, 1999.

[16] P. D. Holm, "A new heuristic UTD diffraction coefficient for nonperfectly conducting wedges," IEEE Transactions on Antennas and Propagation, vol. 48, no. 8, pp. 1211-1219, 2000.

[17] H. M. El-Sallabi, I. T. Rekanos, P. Vainikainen, "A new heuristic diffraction coefficient for lossy dielectric wedges at normal incidence," IEEE Antennas and Wireless Propagation Letters, vol. 1, pp. 165-168, 2002.

[18] H. Wang, T. S. Rappaport, "A parametric formulation of the UTD diffraction coefficient for real-time propagation prediction modeling," IEEE Antennas and Wireless Propagation Letters, vol. 4, pp. 253-257, 2005.

[19] D. N. Schettino, F. J. S. Moreira, C. G. Rego, "Heuristic UTD coefficients for electromagnetic scattering by lossy conducting wedges," Microwave and Optical Technology Letters, vol. 52, no. 12, pp. 2657-2662, 2010.

[20] C. Saeidi, A. Fard, F. Hodjatkashani, "Full Three-Dimensional Radio Wave Propagation Prediction Model," IEEE Transactions on Antennas and Propagation, vol. 60, no. 5, pp. 2462-2471, 2012.

[21] Propagation by diffraction, ITU-R P.526-7, 2001.

[22] T. Tamir, "On radio-wave propagation in forest environment," IEEE Transactions on Antennas and Propagation, vol 15, no. 6, pp. 806-817, 1967.

[23] D. Dence, T. Tamir, "Radio loss of lateral waves in forest environments," Radio Science, vol. 4, no. 4, pp. 307-318, 1969.

[24] T. Kurner, D. J. Cichon, W. Wiesbeck, "The influence of land usage on UHF wave propagation in the receiver near range," IEEE Transactions on Vehicular Technology, vol. 46, no. 3, pp. 739-747, 1997.

[25] S. J. Orfanidis, Electromagnetic Waves and Antennas, 2016. [Online]. Available: http://eceweb1.rutgers.edu/ orfanidi/ewa/.

[26] G. Apaydin, L. Sevgi. Radio Wave Propagation and Parabolic Equation Modeling. Hoboken, USA: Wiley, 2017.

[27] Remcom, Wireless InSite ${ }^{\circledR}$ Propagation Software, 2021. [Online]. Available: https://www.remcom.com/wireless-insiteem-propagation-software\#wireless-insiteem-propagation-features.

[28] P. L. Mcquate, J. M. Harman, M. E. Johnson, A. P. Barsis, "Tabulation of propagation data over irregular terrain in the 230-to-9200-MHz frequency range. Part II: Fritz Peak Receiver Site,” Institute for Telecommunication Sciences, Boulder, CO, ERL 65-ITS 58-2, 1968.

[29] T. G. Farr, P. A. Rosen, E. Caro et al. "The Shuttle Radar Topography Mission,” Reviews of Geophysics, vol. 45, no. 2, 2007.

[30] F. Beauducel. ReadHGT, 2016. [Online]. Available: https://www.mathworks.com/matlabcentral/fileexchange/36379readhgt--import-download-nasa-srtm-data-files---hgt.

[31] The radio refractive index: its formula and refractivity data, ITU-R P.453-12, 2016.

[32] World Atlas of Ground Conductivity. [Online]. Available: http://hamwaves.com/ground/en/. 